“ (C) 2017 IEEE. Personal use of this material is permitted. Permission from IEEE must be obtained for all other uses, in any current or future media, including

reprinting/republishing this material for advertising or promotional purposes, creating new collective works, for resale or redistribution to servers or lists, or reuse of any copyrighted component of this work in other works." 


\title{
Two- and four-way coupling of cohesive poly-disperse particulate foulants on a metal foam fibre immersed in quiescent fluid
}

\author{
Kuruneru, S.T.W. ${ }^{*}$, Sauret, E${ }^{1}$, Saha, S.C. ${ }^{2}$, Gu, YT ${ }^{1}$
}

*First author's e-mail: sahan.kuruneru@ hdr.qut.edu.au

\author{
${ }^{1}$ School of Chemistry, Physics \& Mechanical Engineering, Science and Engineering Faculty, Queensland \\ University of Technology, Brisbane, Queensland, 4011, Australia
}

\begin{abstract}
${ }^{2}$ School of Mechanical and Mechatronic Engineering, Faculty of Engineering and Information Technology, University of Technology Sydney, Ultimo, New South Wales 2007, Australia
\end{abstract}

\begin{abstract}
The ubiquity and complexity of the unsteadiness of fouling and multiphase flows in various engineering systems signify the need to develop advanced numerical methods to study the underlying phenomena of twophase particle-laden fluid flows in heat exchanger systems such as, compact electronics cooling (i.e. heat sinks) and HVAC\&R systems. Fouling is omnipresent in many industries such as power generation, chemical, petroleum, among others. The mechanisms governing fouling coupled with multiphase foulant-laden fluid flow in porous heat exchangers, such as metal foams, are very complex and poorly understood. This investigation forms the basis for addressing the implications of fouling for a myriad of industrial processes. This study will discuss the development of a coupled finite volume method and discrete element method (FVM-DEM) numerical framework to investigate the mechanisms governing particulate fouling in an idealized metal foam heat exchanger. This study resolves four-way and two-way coupled interactions based on poly-disperse cohesive foulants in fluid-saturated foam. The significance stems from the inclusion of cohesiveness between particle-particle and particle-wall contacts which play a decisive role in the foulant aggregation process prevalent in particles with a diameter smaller than $50 \mu \mathrm{m}$. The present results show that the cohesive foulants exhibit strong tendency to aggregate with time and form chain-like projections. A rigid aggregate stack is formed which alters the fluid velocity of the fluid-filled foam. Quantitative analysis of the foulant count and time-averaged aggregate count is discussed. The presented results and the numerical framework could potentially be used to optimize heat exchanger designs by considering operating conditions and foam morphology (i.e. pore diameter, ligament thickness, porosity) that is most susceptible to particulate fouling.
\end{abstract}

Keywords: Metal Foam, CFD-DEM, Fouling, Heat Exchanger Optimization

\section{Introduction}

Fouling in heat exchangers is ubiquitous in a myriad of industries such as chemical, dairy and food processing, power generation, among others. Heat exchanger is constantly subject to fouling over long periods of time, thereby leading to high financial losses, maintenance costs, safety hazards, and reduction in thermal efficiency. Fouling of the heat transfer surfaces in the dairy industry has an adverse effect on the safety and quality of dairy products (Bansal \& Chen, 2006; Visser \& Jeurnink 1997). Process intensification techniques that employ microstructure devices may encounter a decline in the peak operating capacity in the presence of unwanted particulate foulants or non-pure fluid flows (Schoenitz et al., 2015). Membrane bioreactors (MBR) are gaining extensive popularity in wastewater treatment and encounter an average growth rate of $11 \%$ per annum. However, fouling is responsible for a significant portion of the operating costs of this 
state-of-the-art technique (Drews A., 2010). Fouling of preheat exchangers (PHT) for crude oil distillation units (CDU) has a negative impact on the process economics and the environment (Mozdianfard \& Behranvand, 2015). In particular, the U.S. Department of Energy (2006) enunciated the potentially high fuel savings could be achieved by improving existing operating practices and capital equipment in refineries. Particulate fouling in indoor and outdoor environments are responsible for degrading the performance of heat exchangers in heating, ventilation, air-conditioning, and refrigeration systems (HVAC\&R) (Inamdar et al., 2016). Recent technological advancements in electronics cooling have seen an increase in the compactness of heat sink fins (Moore D.A., 2009); however, it is worth mentioning that the dust particles have a detrimental effect on these very small and dense heat sinks. Clearly, fouling is a multifaceted problem encountered in a myriad of industries. The global heat exchanger market is expected to reach US \$ 78 billion by 2020 . Moreover, heat exchanger fouling may play a role in generating 1-2.5\% of the global carbon dioxide emissions while the economic penalties account for about $0.25 \%$ of the gross domestic product (GDP) of industrialized nations (Müller-Steinhagen, H. et al., 2013). Therefore, the development of energy efficient and effective heat exchange technologies is of paramount importance.

Metal foams are a class of disordered porous media that exhibit a number of unique thermo-physical properties such as high surface area to volume ratio, high heat transfer potential, low weight, high strength, and resistance to high temperature and humidity (Han et al., 2012). Open cell metal foams are manufactured by powder metallurgy moulding whereas closed cell metal foams are commonly made by mixing or injecting gas and foaming agent through molten metal (Fraunhofer IFAM, 2015; Krar \& Gill, 2003). The stochastic and highly irregular structure of open-cell metal foams provide excellent thermal management solutions due to the metal foam's inherent ability to induce fluid mixing, promote turbulence, and augment the heat transfer rate. Metal foams are gaining extensive popularity in the research arena and are seen as a viable alternate to fin-based heat exchangers under certain operating conditions (Bhattacharya \& Mahajan, 2002; Chen et al., 2015; Huisseune et al., 2015; Rad et al., 2015; Seyf \& Layeghi 2010). Schampheleire et al. (2013) performed a comparative assessment of the thermal hydraulic performance of aluminium metal foams and louvered fins in HVAC applications. Firstly, they found that louvered fins perform better than 10 PPI metal foams at air velocities greater than $2 \mathrm{~m} / \mathrm{s}$; however, the metal foam is superior to fins in a purely convective flow. Secondly, a brazed foam heat exchanger achieved similar performance results with the louvered fins due to the fact that brazing, a type of bonding method used in foams, significantly increases contact resistance by up to 44\%. It is not known whether a dual fin-foam heat exchanger system exhibits similar performance issues. It is noteworthy that no studies were conducted using aluminium and copper foams of various PPI's, and foam height, which could potentially have higher thermal conductivities that could form the basis for compensating a high contact resistance. Clearly, the use of foams is not perfectly suited for every application. Tongcai et al. (2014) successfully enhanced waste heat recovery in a thermoelectric generator system by up to $83.56 \%$ when using porous metal foams; this achievement will be beneficial in the electric power industry. Metal foam heat sinks are seen as an attractive alternative to fin-based heat sinks for CPUs. In particular, Mahdi et al. (2006) experimentally investigated the thermal performance of aluminium-foam CPU heat exchangers. The thermal resistance was found to be about $70 \%$ lower compared to fin-based CPU exchangers. Li et al. (2016) concluded that porous metals offers a comparatively better performance than solid metals in pin fins in rectangular mini-channels. Sauret, Abdi \& Hooman (2014) used numerical and experimental methods to examine a partly-filled porous metal foam heat exchanger for waste heat recovery applications. Separation regions were identified, and a varying interface velocity is experimentally observed. Yang et al. (2016) integrated experiments, genetic algorithm, and computational fluid dynamics (CFD) to perform optimization studies of porous pin fins in rectangular channels. It was found that the porous pin fin height has a relatively larger influence on pressure drop than the pitch of the pin fins. Furthermore, rearranging the pin fin showed very miniscule change in the overall heat transfer efficiency. Yucel \& Guven (2007) numerically examined cooling performance of heated elements and porous inserts in a parallel plate channel. It was found that the natural convection is the predominant at low-Reynolds number flows, whereas at high-Reynolds number flows, forced convection prevails; moreover, mean Nusselt number increases with increasing Darcy number thereby mitigating the maximum dimensionless temperature of the blocks. Hung et al. (2013) numerically studied the thermal-hydraulic performance of a sintered porous micro-channel heat sink (MCHS) by varying the pumping power, foam porosity, foam geometry, and foam permeability. One of the key findings is that at a pumping power of $0.8 \mathrm{~W}$, a maximum of $76.6 \%$ improvement in cooling performance of the porous MCHS is observed when the foam porosity increases from $60 \%$ to $85 \%$. 
A myriad of the aforesaid studies investigated porous metal foam heat exchangers; however, the existing literature on these fibrous metal foams are based on several major limiting assumptions which raises questions about the veracity of the true potential of metal foam heat exchangers. In particular, most existing studies are purely based on single phase flow (Bai et al., 2011; Della Torre et al., 2014; Ranut et al., 2014; Yongtong et al., 2017; Xu et al., 2011; Ejlali et al., 2009; Kamath et al., 2014; Yang et al., 2013; Lotfizadeh et al., 2015) which is not the norm in passive heat exchangers such as heat sinks or even in energy generation industries (Traore et al. 2015; Kuruneru et al., 2016). Additionally, the existing studies on these fibrous structures are based on foam porosities greater than or equal to $95 \%$ (Bayomy et al., 2016; Diani et al., 2015; Kuruneru et al., 2016; Yucel \& Guven 2007) which are structurally weak, in some cases, provide only moderate increase in heat transfer, and may not be suitable for specific thermal applications (Dukhan 2006). In fact, the existing literature is almost devoid of information, such as single-phase flows, for porous foams of 70-90 \% porosity. Furthermore, few researchers have studied multiphase solid-gas flows and particulate fouling in metal foams of porosities greater than $90 \%$ (Kuruneru et al. 2017; Sauret et al., 2014). However, these multiphase flow studies that assumed mono-disperse foulants, which are based on identical foulant diameter, contrast with the stark reality that heat exchanger fouling comprises foulants of various diameters and densities. Additionally, the investigation of the cohesiveness or stickiness of foulants, which are prevalent in very small particles (i.e. 1 to $20 \mu \mathrm{m}$ ), in porous media, is not established in the literature. Highly cohesive particles could potentially play a role in the fouling process which is related to the cohesion energy density between particle-particle and particle-wall contacts. This could also play an important role in the heat exchanger efficiency. To the best of the authors' knowledge the existing literature is devoid of information pertaining to unsteady multiphase particle-laden fluid flow and particulate fouling in low-porosity fibrous metal foam heat exchangers based on poly-disperse cohesive foulants.

The objective of this work is to develop a coupled Finite Volume Method (FVM) and Discrete Element Method (DEM) numerical method to examine the transient evolution of multiphase foulant-laden air flow and poly-disperse cohesive particulate fouling and aggregation in low-porosity fibrous foams. In this work, two-way coupling (particle-fluid interactions) and four-way coupling (particle-particle and particle-wall interactions) is taken into account. This paper is organized as follows. First, we cover the numerical methodology in $\S 2.1$. The computation domain, mesh, and boundary conditions are discussed in $\S 2.2$. An outline of the numerical cases studies based on various foulant properties is presented in $\S 2.3$. The main results covering grid independence test, model validation, and key findings of solid-gas flows are discussed in $\S 3$. Finally, the conclusions drawn from the main results are given in $\S 4$.

\section{Methodology}

\subsection{Numerical Model}

The study of multiphase particle-laden fluid flow and particulate fouling in a porous structure is achieved by developing a coupled finite-volume method (FVM) and discrete-element method (DEM). Herein is presented a summary of the numerical framework to account for fully resolved solid-gas non-reactive interactions in a porous configuration consisting of arrays of circular cylindrical obstructions. The full details of the deployed numerics is presented in Kuruneru et al. (2017), and is not repeated here. The working fluid in this study is air which is assumed to be laminar, incompressible, and isothermal and governed by the volume-averaged Navier-Stokes equations:

$$
\begin{aligned}
& \frac{\partial \varepsilon_{f}}{\partial t}+\nabla \cdot\left(\varepsilon_{f} v_{f}\right)=0, \\
& \frac{\partial\left(\rho_{f} \varepsilon_{f} v_{f}\right)}{\partial t}+\nabla \cdot\left(\rho_{f} \varepsilon_{f} v_{f} v_{f}\right)=-\varepsilon_{f} \nabla p+F_{p f}+\nabla \cdot\left(\varepsilon_{f} \tau\right)+\rho_{f} \varepsilon_{f} g,
\end{aligned}
$$

where $v_{f}$ is the fluid velocity, $\varepsilon_{f}$ is the gas-phase fraction within a finite-volume computation cell which accounts for the fraction of solid particles in every computational cell, $g$ is gravitational acceleration, $\tau$ is the fluid viscous stress tensor, and $\rho_{f}$ is the carrier fluid density. The two-way coupling interaction term which 
accounts for the momentum transfer between the discrete solid particles (hereinafter called "foulants") and air is given by $F_{p f}$,

$$
F_{p f}=\frac{1}{V_{\text {cell }}} \sum_{\forall p \in \text { cell }} \frac{V_{i} \eta}{1-\varepsilon_{f}}\left(v_{i}-v_{f \mid i}\right)
$$

where $v_{i}$ is the particle velocity, $V_{i}$ is the particle volume, and $V_{\text {cell }}$ is the volume of a computational cell for all particles $I, v_{i}$ is the solid foulant velocity, and $v_{f \mid i}$ is the air velocity interpolated to the position of the foulant. In this study, the inter-phase momentum exchange term $\eta$ is based on the drag closure formulation of Gidaspow, which is commonly referred to as the Ergun-Wen-Yu drag model (Gidaspow, 1994). Secondly, the transportation of the discrete solid foulants is governed by Newton's second law of motion:

$$
m_{i} \frac{d v_{i}}{d t}=m_{i} g+F_{\text {contact }}+\frac{V_{i} \eta}{1-\varepsilon_{f}}\left(v_{f \mid i}-v_{i}\right)
$$

The evaluation of the angular momentum of a discrete particle is given by

$$
I_{i} \frac{d \omega_{i}}{d t}=T_{i}
$$

where $\omega_{i}$ is the rotational velocity, $T_{i}$ is the torque, and $I_{i}$ is the moment of inertia. An Euler-implicit integration scheme is deployed for the particle equations. The discrete particles are assumed to be spherical, smooth, rigid, and isothermal. The density of solid foulant $\rho_{i}$ is assumed to be significantly greater than the density of air $\rho_{f}$ $\left(\rho_{i} \gg \rho_{f}\right)$; as such, only drag forces and gravity force is considered. A soft-sphere discrete element method (DEM) is deployed in this study which is based on a non-linear spring-slider-dashpot model and is used to model the discrete particulate phase (Tsuji et al., 1992). The interested reader is referred to Tu et al.(2012) for a summary of the linear and non-linear contact models for DEM simulations.

A cohesion model is introduced to account for cohesiveness between particle-particle and particle-wall contacts (DEM Solutions, 2010b; Qian et al., 2013; Romani Fernandez \& Nirschl 2013). The cohesive force accounts for the cohesive energy density of the solid particles $\chi$ and the contact between the particles $A_{\text {contact }}$, given by:

$$
F_{C E D}=\chi A_{\text {contact }}
$$

The number of collision resolution steps is set to 30 and the time-step is set to $1 \times 10^{-4}$ for the fluid phase. The simulation is executed from $0 \mathrm{~s}$ to $1.00 \mathrm{~s}$ and the solid particles (i.e. foulants) are injected at the inlet plane from $0.10 \mathrm{~s}$ to $1.00 \mathrm{~s}$. The fluid is quiescent, in other words, $U_{\text {inlet }}=0 \mathrm{~m} / \mathrm{s}$. Particle initialization velocity is set to $0 \mathrm{~m} / \mathrm{s}$. A generalized geometric-algebraic multi-grid (GAMG) solver and a Gauss-Seidel smoother is used to obtain the discretised pressure equations. Additionally, a bi-conjugate gradient stabilized (PBiCGStab) with a diagonal incomplete-Cholesky symmetric and incomplete-LU asymmetric preconditioner (DILU) is deployed to obtain discretised velocity equations. The PBiCGStab is a variant of the biconjugate gradient method (BiCG) (Van der Vorst, 1992; Barrett et al., 1994). Trial numerical results have shown that the results are very similar regardless of whether a smoother symmetric Gauss-Seidel (sGS) or a PBiCGStab-DILU is used for obtaining the discretised velocity equations. The residuals for the pressure and velocity are, respectively, $1 \times 10^{-6}$ and $1 \times 10^{-5}$. The density of the solid sandstone particles is $1250 \mathrm{~kg} / \mathrm{m}^{3}$ (Hilton \& Cleary 
2013). In this study, the coefficient of friction is assumed to be, respectively, 0.50 and 0.30 for particle-particle contacts and particle-wall contacts. For particle-wall contacts, the particle is assumed to adhere upon impact on the surface (metal foam ligament); in other words, the particle-wall collisions are assumed to be perfectly inelastic. The restitution coefficient for particle-particle is 0.50 . This assumption based on $100 \%$ sticking probability for various heat transfer equipment, such as HVAC\&R, is used by various authors (Inamdar et al., 2016; Muyshondt et al., 1998, Siegel 2002, Siegel \& Nazaroff, 2003; Yang et al., 2012). The cohesion energy density and adhesion energy density between the particle-particle and particle-wall contacts is, respectively, $50000 \mathrm{~J} / \mathrm{m}^{3}$ (Qian et al., 2013). The solid-gas flow profiles and particulate fouling deposition characteristics based on various particle injection rates are studied. The enabling of the momentum predictor showed negligible difference in the numerical results and is usually not enabled for (very) low-Reynolds number fluid flows or multiphase flows. A dual SIMPLE-PISO (PIMPLE) algorithm is deployed for the fluid flow to achieve a stable pressure-velocity coupling and is used in conjunction with the discrete particulate phase. Several key controls are introduced: non-orthogonal correctors, correctors, outer-correctors, relaxation factors, and a PIMPLE residual control. Numerical sensitivity analysis study showed minuscule discrepancy in the results using either the standalone PISO or dual SIMPLE-PISO algorithm. OpenFOAM software (OpenFOAM v4.1), an open-source customizable CFD C++ program, is used to numerically couple the carrier fluid phase and discrete phase.

\subsection{Geometry}

The developed numerical framework is deployed to study solid-gas multiphase laminar flows and particulate fouling in a porous structure which consists of a cubical array of cylindrical struts, as shown in Fig. 1. Lowporosity foams have a more circular ligament shape (Bhattacharya et al., 2002). The dimensions of the geometry is $0.50 \mathrm{~mm} \times 0.50 \mathrm{~mm}$. The circular cylindrical obstructions which connote the metal foam ligaments have a diameter of $0.175 \mathrm{~mm}$. A CFD-DEM analysis of particle-laden gas flows in an actual metal foam geometry obtained through X-ray micro CT scans is extremely complex and computationally demanding. As such, the first step is of the authors' intention to simulate a simplified and repeated configuration while retaining the macroscopic quantities of a metal foam such as porosity and fibre thickness. Many authors evaluated heat transfer correlations for high-porosity aluminium foams based on the assumption that the foams form an idealized pattern, namely, a cylindrical array of cylinders (Dukhan 2005; Fuller et al., 2005; Ghosh 2009; Hooman et al., 2012; Tamayol \& Bahrami 2011), as shown in Fig.1 for example. In particular, Wang et al. (2017) used a cylindrical array of two cylinders to numerically model fouling characteristics of H-type heat exchangers. It is of the authors' intention to unravel the dynamics of solid-gas flows and fouling in an idealized model of cylindrical circular arrays prior to studying real metal foam structures. This idealized geometry consists of multiple ordered circular cylindrical arrays; however, in this study, only one ligament (cylinder) is studied due to the symmetrical nature of the problem. Moreoever, the focus is on the investigation on the cohesive behaviour of foulants at the pore-level. As such, one 2D cylindrical array is selected as the computational domain. A velocity inlet, $U_{\infty}=0 \mathrm{~m} / \mathrm{s}$, is assigned at the top plane, and a pressure outlet $(0 \mathrm{~Pa})$ is assigned at the bottom plane; additionally, symmetry boundary conditions are applied to the left and the right planes, as shown in Fig. 1. It is assumed that symmetry left and right planes are permeable to the solid foulant particles. The geometry and the corresponding hexahedron mesh in the form of quadrilateral grids is designed in ANSYS (ANSYS v18, 2016). The simulation is executed in OpenFOAM (OpenFOAM v4.1, 2016). ParaView (ParaView v4.1.0, 2014) is used to post-process the numerical results. The porosity of a metal foam is approximated by,

$$
\varepsilon=1-\frac{3 \pi}{4}\left(\frac{d_{f}}{d_{p}}\right)^{2},
$$

Manuscript prepared for: International Communications in Heat and Mass Transfer 
where $d_{f}$ and $d_{p}$ are, respectively, the ligament diameter and the pore diameter. A foam porosity $\varepsilon$, based on a ligament diameter of $0.175 \mathrm{~mm}$ and pore diameter of $0.50 \mathrm{~mm}$, of $71.15 \%$ is registered. The number of pores per inch (PPI) in this study is 52. The PPI is evaluated as:

$$
d_{f}=\left(0.431-0.0049 P P I+\frac{2.43}{P P I^{2}}\right) / 1000
$$

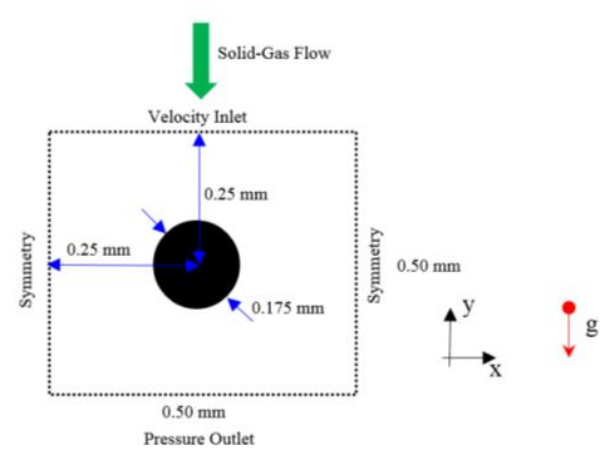

(a)

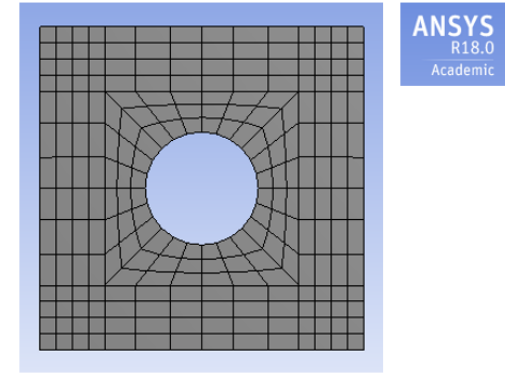

(b)

Figure 1. (a) Computation domain and boundary conditions (b) Computational grid.

A grid independent test on single-phase fluid flow is performed using three different grids: 272 nodes (which is based on Fig. 1b), 480 nodes, 744 nodes. Mesh is refined using the sizing option, namely the relevance centre, smoothing, span angle centre. Successive mesh refinement shows no alteration in the global pressure drop at 272 nodes. This is arguably due to the simplicity of the domain. Therefore, Grid 1 is used for the computation studies. It is worth mentioning that the use of the unresolved CFD-DEM numerical method presented herein precludes the use of extremely fine fluid grid cells relative to the size of the DEM particles, which could lead to numerical stability and accuracy issues (Banerjee \& Agarwal 2015; Kloss et al., 2012). As such, the mesh at the wall is not refined. The use of the resolved CFD-DEM method which is based on the fictitious domain method is to be used if the DEM particle is significantly greater the CFD mesh cell (i.e. extremely fine mesh) or when dealing with complex geometries (Hager et al., 2012).

\subsection{Case Studies}

The numerical investigation is based on poly-disperse solid cohesive foulant particles, as shown in Table 1. The poly-disperse solid foulants are injected at the inlet plane based on a normal Gaussian size distribution with a minimum particle diameter of $5 \mu \mathrm{m}$, maximum diameter of $20 \mu \mathrm{m}$, mean diameter of $10 \mu \mathrm{m}$, and variance of 25. The dust diameter and densities are based on the American Society of Heating, Refrigerating and Air-Conditioning Engineers (ASHRAE) standard test dust (Inamdar et al., 2016). Furthermore, the particulate fouling patterns is investigated based on various particle injection rates, which is based on the total number of particles injected per second (pps) at the inlet plate.

Table 1 CFD-DEM case studies.

\begin{tabular}{ccc}
\hline Case & Foulant density $\left(\mathbf{k g} / \mathbf{m}^{\mathbf{3}}\right)$ & Particle Injection Rate (pps) \\
\hline $1 \mathrm{~A}, 1 \mathrm{~B}, 1 \mathrm{C}$ & $1250,500,2500$ & 50 \\
$2 \mathrm{~A}, 2 \mathrm{~B}, 2 \mathrm{C}$ & $1250,500,2500$ & 150
\end{tabular}




$\begin{array}{lll}3 \mathrm{~A}, 3 \mathrm{~B}, 3 \mathrm{C} & 1250,500,2500 & 250 \\ 4 \mathrm{~A}, 4 \mathrm{~B}, 4 \mathrm{C} & 1250,500,2500 & 350\end{array}$

Firstly, the solid-gas flows and foulant aggregation and foulant deposition characteristics based on solid particles of $1250 \mathrm{~kg} / \mathrm{m}^{3}$ density is investigated. Afterwards, the foulant aggregation profiles based on a lower density $\left(500 \mathrm{~kg} / \mathrm{m}^{3}\right)$ and higher density $\left(2500 \mathrm{~kg} / \mathrm{m}^{3}\right)$ is investigated. Moreover, we examine the solid aggregation profiles based on various particle injection rates or the number of particles injected into the domain per second (pps).

\section{Results \& Discussions}

\subsection{Numerical Model Validation}

The bouncing motion of a solid particle onto a glass wall filled with air is used as a benchmark for validating the numerical model. Initially, the particle, at a specified height from the glass wall, is at rest together with the ambient fluid. At the start of the simulation, the particle is released from rest and settles to the bottom wall under the action of gravity. The particle then bounces off the bottom wall and attains a specific height at time $t$.
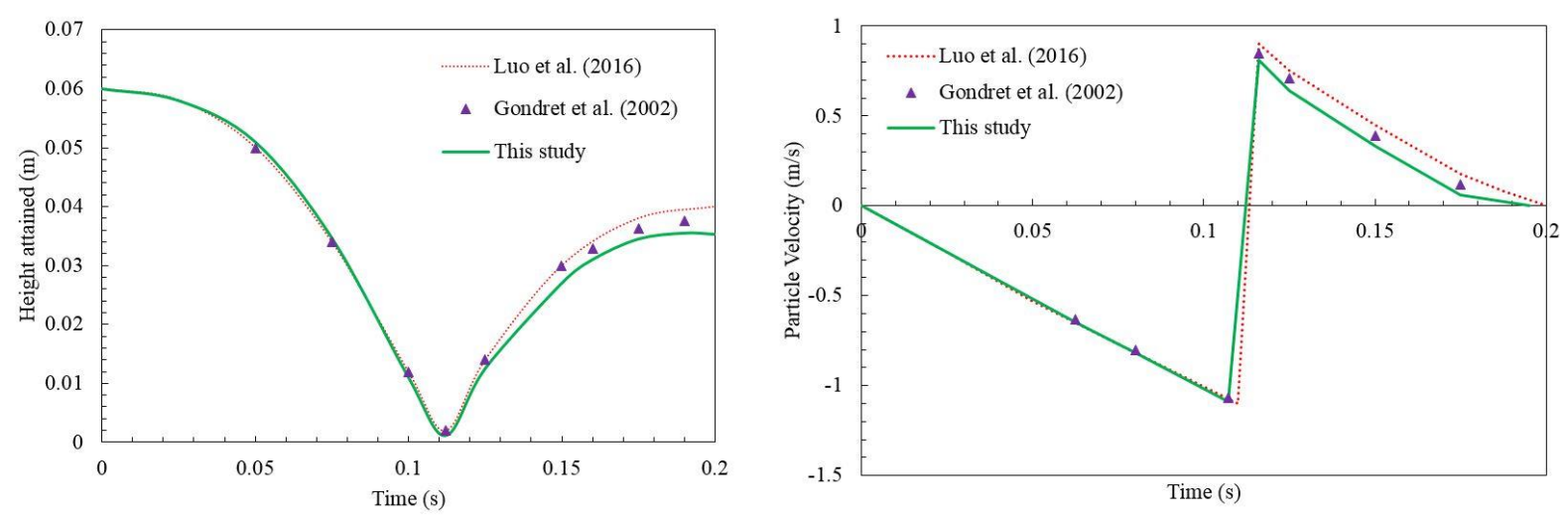

Figure 2. Comparison of present results against numerical results from Luo et al. (2016) and experimental data from Gondret et al. (2002): (a) height attained by particle (b) particle velocity

The solid particle is based on a $0.006 \mathrm{~m}$ teflon bead impacting a soda glass wall enclosed in a $120 \mathrm{~mm} \times 120$ $\mathrm{mm} \times 120 \mathrm{~mm}$ cuboid. The particle density is $2150 \mathrm{~kg} / \mathrm{m}^{3}$. The quiescent fluid is air with a density of 1.225 $\mathrm{kg} / \mathrm{m}^{3}$ and kinematic viscosity of $1.6 \times 10^{-5} \mathrm{~m}^{2} / \mathrm{s}$. The particle is placed at the centre of the cuboid without any particle initialization velocity. The mesh cell size to particle diameter ratio is $2.5: 1$. The restitution coefficient and friction is set to 0.80 and 0 respectively. The numerical results are compared against the experimental findings of Gondret et. al. (2002) and numerical results of Luo et al. (2016). The maximum height the teflon ball attains at a specified time point is shown in Fig. 2a and the particle's velocity corresponding to the particle's height attained is shown in Fig. 2b. Good agreement is observed between the present numerical results and the findings by Luo et al. (2016) and Gondret et al. (2002) thus lending credence to the numerical model. 


\subsection{Solid-Gas Flow and Fouling Profile}

The time series of foulant-laden air flow and particulate deposition and particulate aggregation pertaining to $1250 \mathrm{~kg} / \mathrm{m}^{3}$ foulants at $250 \mathrm{pps}$ is shown in Fig. 3. Particle injection commences at $0.100 \mathrm{~s}$. The interactions between the neighbouring foulants and particle-wall interactions (four-way coupling) and particle-fluid interactions (two-way coupling) are resolved. As time elapses, the foulants traverse in the quiescent fluid towards the outlet by means of gravitational sedimentation. Traces of foulants sediment on the surface of the circular obstruction (ligaments), and a number of the solids adhere to the region surrounding the apex of the upper half of the cylinder. However, the particles that settle at or near the apex of the upper half of the cylinder eventually slide over and detach from the cylindrical surface. This is because the incoming particles impact the settled particles in that region thereby unshackling any aggregate matter causing them to slide and attain a non-zero velocity. The influence of solid foulants on the fluid velocity field is realized; the fluid velocity remains constant bearing $0 \mathrm{~m} / \mathrm{s}$ from $0 \mathrm{~s}$ to $0.100 \mathrm{~s}$ whereas the fluid velocity profiles vary with time due to the presence of solid foulants. The foulant aggregate comprises a semi-rigid structure of multiple layers of stationary foulants stacked together forming chain-like projections some of which are attached to the wall and some are away from the walls, which is clearly observed in Fig. 3f as a typical example. These chain-like or caterpillar-like projections are clearly denoted by the dashed circular lines shown in Fig. 3f, say. It is worth mentioning that Mousel \& Marshall (2010) observed similar phenomenon pertaining to the formation of chainlike adhesive aggregates through a micro-nozzle. The particle size in their study is $20 \mu \mathrm{m}$. The evenness of the foulant aggregate height on the cylinder is clearly observed throughout the simulation; however, the aggregate height amplifies from $0.500 \mathrm{~s}$ and reaches its peak at $0.600 \mathrm{~s}$. The aggregate clearly projects outwards away from the cylinder at $0.600 \mathrm{~s}$ before becoming unstable and collapses due to the aggregate weight which is observed at $0.700 \mathrm{~s}$.

The foulants adhere to the circular walls thereby artificially increasing the surface roughness of the cylindrical ligaments. Moreover, the continuous accumulation of particles ultimately alters the porosity of the configuration. This could potentially reduce the overall heat transfer performance of the porous structure depending on the thermal conductivity of the solid particles and the circular ligament walls. The velocity of the foulants increase upon reaching the symmetric walls because zero normal gradient is enforced for any variable apart from the normal velocity which is zero.

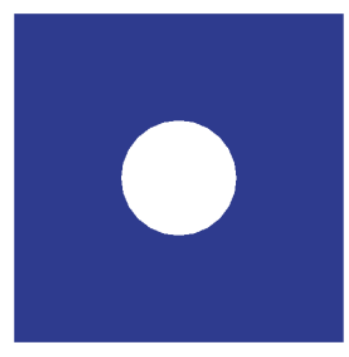

(a) $t=0.10 \mathrm{~s}$

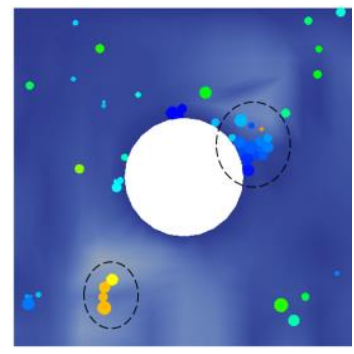

(f) $t=0.60 \mathrm{~s}$

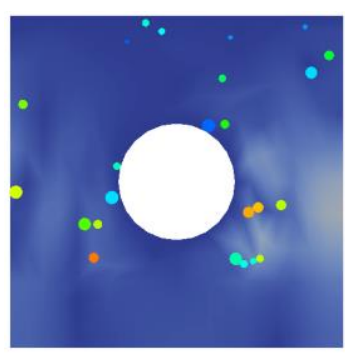

(b) $t=0.20 \mathrm{~s}$

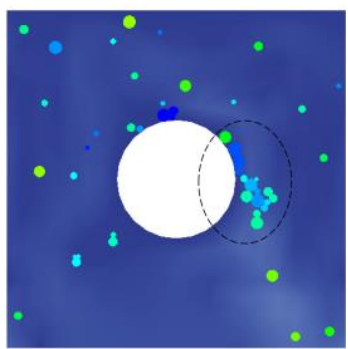

(g) $t=0.70 \mathrm{~s}$

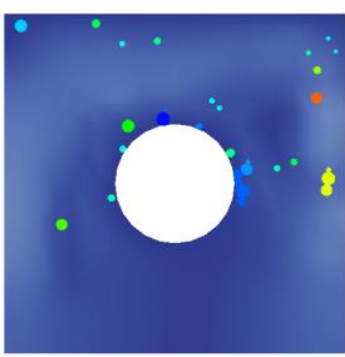

(c) $t=0.30 \mathrm{~s}$

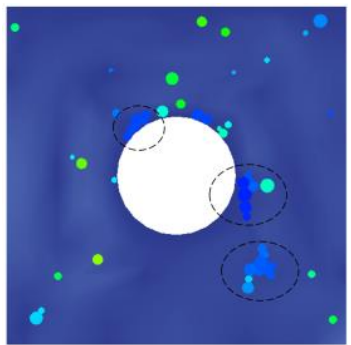

(h) $t=0.80 \mathrm{~s}$

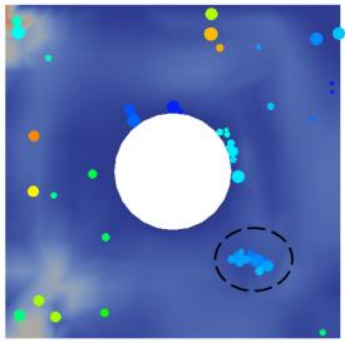

(d) $t=0.40 \mathrm{~s}$

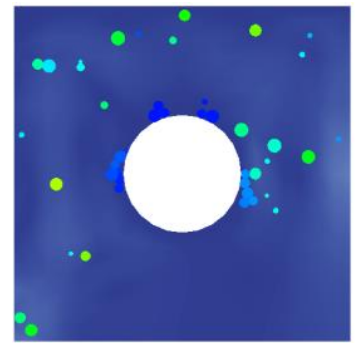

(i) $t=0.90 \mathrm{~s}$

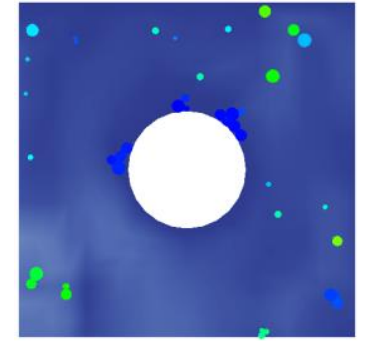

(e) $t=0.50 \mathrm{~s}$

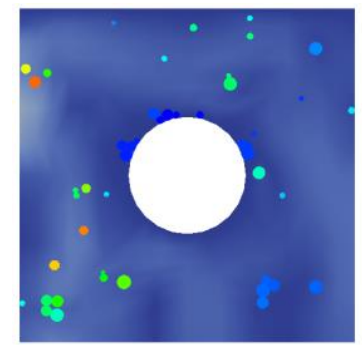

(j) $t=1.00 \mathrm{~s}$

Manuscript prepared for: International Communications in Heat and Mass Transfer 


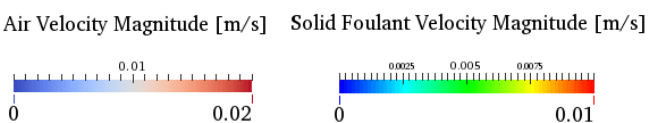

Figure 3. Time evolution of foulant-gas flows and foulant aggregation of $1250 \mathrm{~kg} / \mathrm{m}^{3}$ foulants at $250 \mathrm{pps}$.

Traces of particles of various diameters are consistently scattered in the system throughout the entire simulation, and although a number of particles exit the domain through the outlet, few particles remain in the system for some time forming aggregates. The cohesive forces are capable of binding the solid particles into chains of aggregates such as those observed in Fig. 3f, 3g, 3h. Numerical simulations have shown that the $1250 \mathrm{~kg} / \mathrm{m}^{3}$ particles has shown no aggregate formation at $50 \mathrm{pps}$. However, it is noteworthy that although the number of aggregates remain invariant, for example, from 0.25 to 0.45 , the total number of particles attached to each of the aggregates differs substantially. For examples, at $0.550 \mathrm{~s}$, one aggregate comprises a maximum of 12 attached particles, whereas at $0.250 \mathrm{~s}$, there is a maximum of three attached particles in one aggregate.

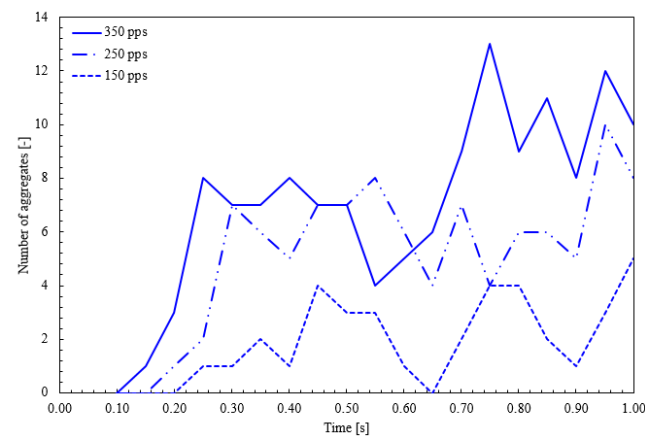

(a)

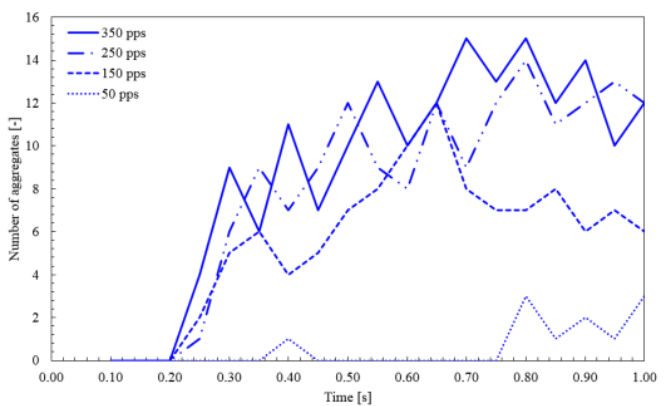

(c)

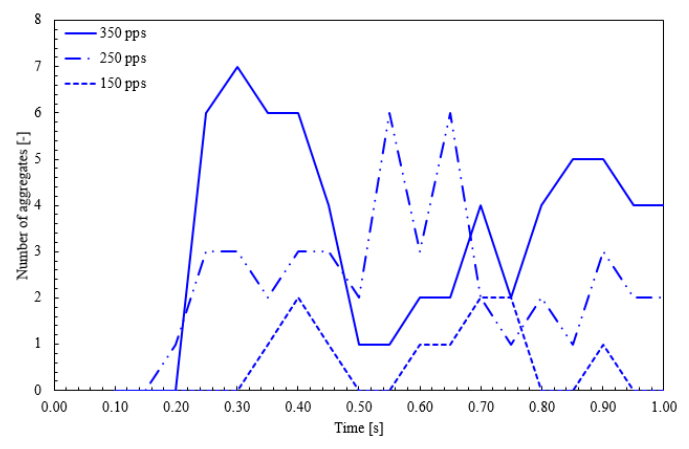

(b)

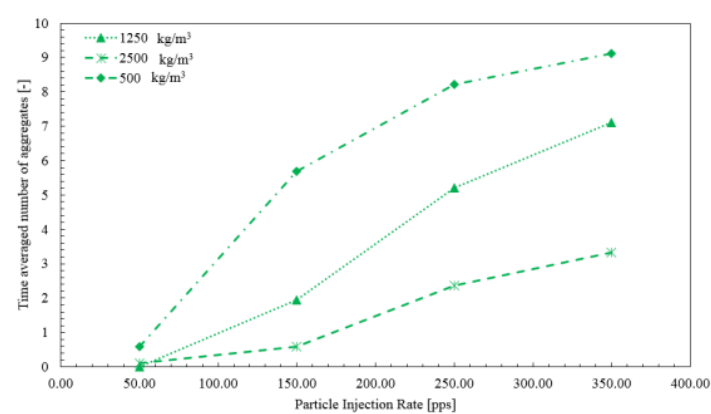

(d)

Figure 4. Number of aggregates (a) $1250 \mathrm{~kg} / \mathrm{m}^{3}$ foulants (b) $2500 \mathrm{~kg} / \mathrm{m}^{3}$ foulants (c) $500 \mathrm{~kg} / \mathrm{m}^{3}$ foulants (d) time-averaged aggregate quantity

The time evolution of the foulant aggregate quantity is shown in Fig. 4. The $1250 \mathrm{~kg} / \mathrm{m}^{3}$ and $2500 \mathrm{~kg} / \mathrm{m}^{3}$ foulant particles do not exhibit any aggregation process at $50 \mathrm{pps}$; however, traces of aggregation of $500 \mathrm{~kg} / \mathrm{m}^{3}$ particles is profound at certain time-points at $50 \mathrm{pps}$, as shown in Fig. 4. The reason is because the heavier particles, namely $2500 \mathrm{~kg} / \mathrm{m}^{3}$, settle to the outlet plane rapidly, whereas the lighter $500 \mathrm{~kg} / \mathrm{m} 3$ particles settle at a comparatively slower pace which promotes a greater probability for these particles to aggregate. The number of particles present in the system from $0 \mathrm{~s}$ to $1 \mathrm{~s}$ is shown in Fig. 5. A high particle injection rates leads to an increase in the number of particles in the system thereby increasing the chances of the occurrence of particle aggregation at the cylinder surface or away from the cylinder surface. The time-averaged quantity of foulants based on four different injection rates is shown in Fig. 6. According to Fig. 6, the $1250 \mathrm{~kg} / \mathrm{m}^{3}$ and Manuscript prepared for: International Communications in Heat and Mass Transfer 
$500 \mathrm{~kg} / \mathrm{m}^{3}$ show a steady increase in the number of particles with time at 350 pps but quantity of the heavier $2500 \mathrm{~kg} / \mathrm{m}^{3}$ foulants remain resolutely steady commencing $0.50 \mathrm{~s}$. However, all particle densities do not show any significant change in the number of particles from $0.20-0.30 \mathrm{~s}$ at $50 \mathrm{pps}$ and $150 \mathrm{pps}$. All three particle densities exhibit a linear increase in the time-averaged number of particles with increasing particle injection rate. Several cases have shown traces of chain deposition phenomenon where incoming particles impact the wall-attached foulants and causes the aggregate height to increase to a certain extent. The maximum height at which an aggregate achieves under this phenomenon largely depends on the fluid flow conditions and particle properties. This chain deposition phenomenon is prevalent in a number of the presented studies, such as Fig. 3 and Fig. 5. The lower half of the cylinder represents a shadow like region where particle-wall cohesion is scarcely observed for very few particles such as $5 \mu \mathrm{m}$. The few particles that deposit in this region ultimately detach from the cylinder walls due to the collision by larger individual particles or groups of particles in the form of aggregates.

Another interesting phenomenon is shown in Fig.5. After the aggregate slides towards the apex of the midsection of the cylinder, the aggregate eventually detaches from the cylinder surface while retaining the cohesive bonded aggregate structure, as shown by the dashed circle in Fig. 5b. Afterwards, the aggregate continues to remain chained as a single aggregate from the moment it leaves the cylinder to the time it slowly drifts towards the outlet plane, as shown in Fig 5c. Another aggregate is formed in a similar fashion denoted by the bold circle in Fig. 5. This chain aggregation phenomenon due to the presence of the wall is notably prevalent in a number of cases. The break-away conditions of aggregate bonds, length of aggregate, and the duration of aggregates is linked to the competition between the solid-solid and solid-wall cohesive forces and hydrodynamic solid-gas interactions. An increase in the particle injection rate has a greater tendency to increase aggregate numbers and aggregate process. However, the larger injection rates have a higher frequency of particle-particle collisions and the incoming particles or neighbouring particles could impact and dismantle the large chain aggregates. In other words, it can be inferred that the large chain aggregates have a greater tendency to be broken off to smaller aggregates due to the higher collision frequency between incoming particles or neighbouring particles or aggregates.

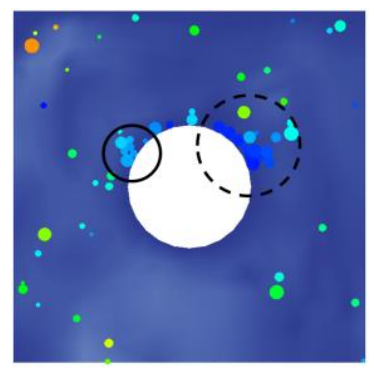

(a) $\mathrm{t}=0.600 \mathrm{~s}$

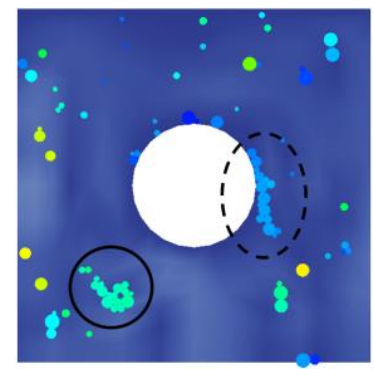

(b) $t=0.750 \mathrm{~s}$

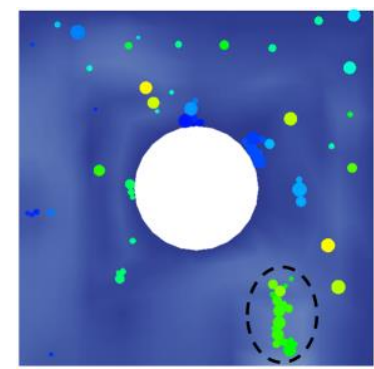

(c) $\mathrm{t}=0.820 \mathrm{~s}$

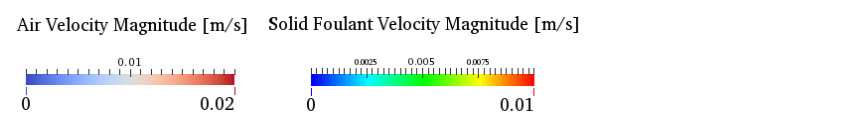

Figure 5. Aggregate profile of $1250 \mathrm{~kg} / \mathrm{m}^{3}$ foulants at $350 \mathrm{pps}$ at various time-points 


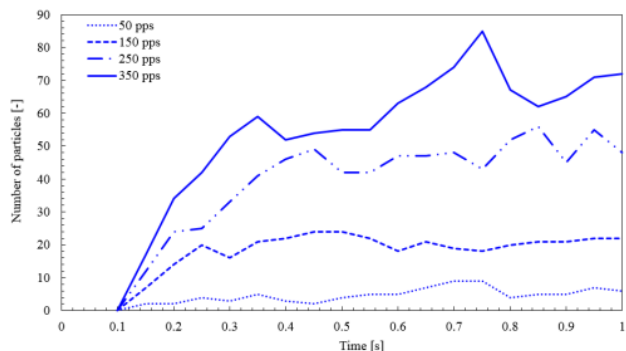

(a)

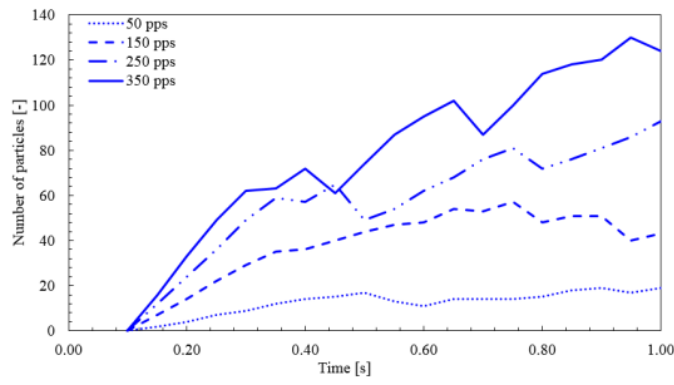

(c)

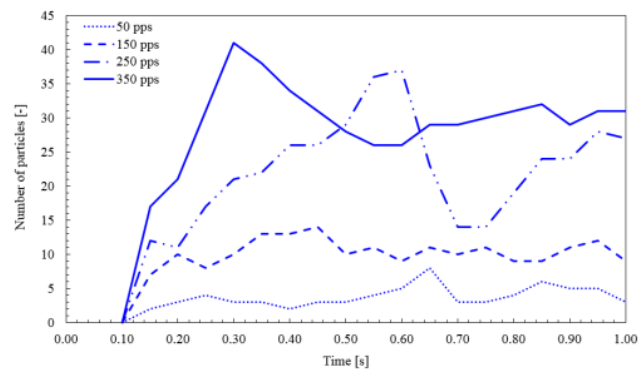

(b)

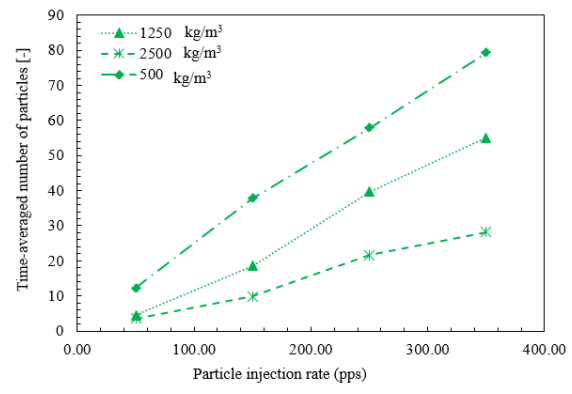

(d)

Figure 6. Number of particles in system (a) $1250 \mathrm{~kg} / \mathrm{m}^{3}$ foulants (b) $2500 \mathrm{~kg} / \mathrm{m}^{3}$ foulants (c) $500 \mathrm{~kg} / \mathrm{m}^{3}$ foulants (d) time-averaged foulant quantity

The presence of the cylinder is primarily responsible for the creation of very large chain aggregates. The heavier $2500 \mathrm{~kg} / \mathrm{m}^{3}$ exhibit a lower tally of aggregates. The lighter $500 \mathrm{~kg} / \mathrm{m} 3$ particles have a higher residence time thereby creating a higher likelihood for particles to collide with each other; moreover, this long aggregate structure captures some of the incoming particles from the inlet, as shown in Fig. 7. According to Fig. 4, the $2500 \mathrm{~kg} / \mathrm{m}^{3}$ particles exhibit the least number of aggregates. The smaller foulants exhibit stronger adhesiveness; the followability of these particles are slightly lower than the heavier particles. Interestingly, traces of highly conductive foulants, such as copper dust, could potentially accentuate heat transfer performance in certain applications. This could play a role in the overall heat transfer performance of a porous material.

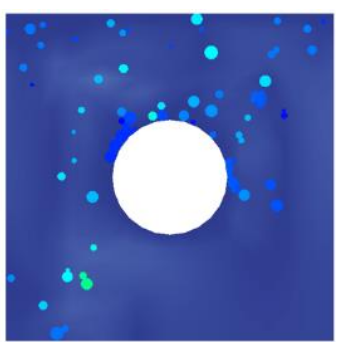

(a) $\mathrm{t}=0.600 \mathrm{~s}$

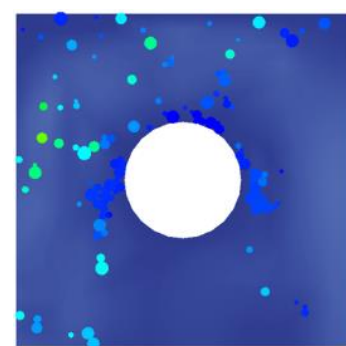

(b) $\mathrm{t}=0.800 \mathrm{~s}$

Figure 7. Aggregate profile of $500 \mathrm{~kg} / \mathrm{m}^{3}$ foulants at $350 \mathrm{pps}$ at $0.600 \mathrm{~s}$ and $0.800 \mathrm{~s}$.

\section{Conclusions}

A coupled finite-volume (FVM) and discrete element (DEM) is developed to numerically examine the transient nature of cohesive poly-disperse foulant-laden air flow, foulant aggregation, and particulate fouling in a cylindrical array. A comparative assessment of the solid-gas profiles based on various foulant densities and foulant injection rates are investigated. The particle diameter in this study is less than $20 \mu \mathrm{m}$ which necessitates the deployment of a model that accounts for cohesiveness of particle-particle and particle-wall 
contacts. As such, the crucial aspect of this study is the inclusion of the cohesion model to account for the cohesive behaviour of foulants. The simulation considers two-way and four-way interactions between the solid foulants, the viscous fluid, and circular ligament wall. The time-averaged aggregate count increases with time regardless of the injection rate and foulant density. Smaller particles are shown to exhibit stronger cohesiveness and remain in an aggregate for substantial time. Heavier particles aggregate at certain timepoints, but will break off upon impact with an incoming single particle or incoming neighbouring aggregate. Importantly, the presented numerical model permits one to better optimize compact and light-weight heat exchanger designs by altering foam geometrical properties by taking into account regions of high concentration of foulants. In the next step, the authors will investigate non-isothermal solid-gas flows and thermal effects on the aggregation process.

\section{Acknowledgements}

Kuruneru, S.T.W. acknowledges the financial support in the form of the Australian Postgraduate Award (APA) scholarship granted by the Australian government. The authors gratefully acknowledge the computational resources provided by QUT's High Performance Centre (HPC).

\section{Conflict of Interest}

None declared.

\section{References}

ANSYS $®$ Academic Research, Release 18.0 (2016).

Bai, M., \& Chung, J. N. (2011). Analytical and numerical prediction of heat transfer and pressure drop in open-cell metal foams. International Journal of Thermal Sciences, 50(6), 869-880.

Banerjee, S., \& Agarwal, R. K. (2015). Transient reacting flow simulation of spouted fluidized bed for coaldirect chemical looping combustion. Journal of Thermal Science and Engineering Applications, 7(2), 021016-021016-9.

Bansal, B., \& Chen, X. D. (2006). A critical review of milk fouling in heat exchangers. Comprehensive Reviews in Food Science and Food Safety, 5(2), 27-33.

Bayomy, A., Saghir, M., \& Yousefi, T. (2016). Electronic cooling using water flow in aluminum metal foam heat sink: Experimental and numerical approach. International Journal of Thermal Sciences, 109, 182-200.

Bhattacharya, A., \& Mahajan, R. L. (2002). Finned metal foam heat sinks for electronics cooling in forced convection. Journal of Electronic Packaging, Transactions of the American Society of Mechanical Engineers, 124(3), 155-163.

Bhattacharya, A., Calmidi, V. V., \& Mahajan, R. L. (2002). Thermophysical properties of high porosity metal foams. International Journal of Heat and Mass Transfer, 45(5), 1017-1031.

Chen, X., Tavakkoli, F., \& Vafai, K. (2015). Analysis and characterization of metal foam-filled double-pipe heat exchangers. Numerical Heat Transfer, Part A: Applications, 68(10), 1031-1049.

Manuscript prepared for: International Communications in Heat and Mass Transfer 
Della Torre, A., Montenegro, G., Tabor, G., \& Wears, M. (2014). CFD characterization of flow regimes inside open cell foam substrates. International Journal of Heat and Fluid Flow, 50, 72-82.

DEM Solutions, L., 2010b. EDEM 2.3 User Guide-Appendix A: Contact model theory.

Diani, A., Bodla, K. K., Rossetto, L., \& Garimella, S. V. (2015). Numerical investigation of pressure drop and heat transfer through reconstructed metal foams and comparison against experiments. International Journal of Heat and Mass Transfer, 88, 508-515.

DOE. Energy bandwidth for petroleum refining processes; U.S. Department of Energy, Office of Energy Efficiency and Renewable Energy Industrial Technologies Program, 2006.

Drews, A. (2010). Membrane fouling in membrane bioreactors - Characterisation, contradictions, cause and cures. Journal of Membrane Science, 363(1), 1-28.

Dukhan, N., Quiñones-Ramos, P. D., Cruz-Ruiz, E., Vélez-Reyes, M., \& Scott, E. P. (2005). Onedimensional heat transfer analysis in open-cell 10-ppi metal foam. International Journal of Heat and Mass Transfer, 48(25), 5112-5120.

Dukhan, N. (2006). Correlations for the pressure drop for flow through metal foam. Experiments in Fluids, 41(4), 665-672.

Ejlali, A., Ejlali, A., Hooman, K., \& Gurgenci, H. (2009). Application of high porosity metal foams as aircooled heat exchangers to high heat load removal systems. International Communications in Heat and Mass Transfer, 36(7), 674-679.

Fraunhofer IFAM. (2015). Retrieved from

http://www.ifam.fraunhofer.de/en/Bremen/Formgebung_Funktionswerkstoffe/Leichtbauwerkstoffe/Metallsc haeume/Herstellungsverfahren.html

Fuller, A. J., Kim, T., Hodson, H. P., \& Lu, T. J. (2005). Measurement and interpretation of the heat transfer coefficients of metal foams. Proceedings of the Institution of Mechanical Engineers, Part C: Journal of Mechanical Engineering Science, 219(2), 183-191.

Ghosh, I. (2009). Heat transfer correlation for high-porosity open-cell foam. International Journal of Heat and Mass Transfer, 52(5), 1488-1494.

Gidaspow, D. (1994). Multiphase flow and fluidization: continuum and kinetic theory descriptions. Academic press.

Gondret, P., Lance, M., \& Petit, L. (2002). Bouncing motion of spherical particles in fluids. Physics of Fluids, 14(2), 643-652.

Hager, A., Kloss, C., Pirker, S., \& Goniva, C. (2012). Parallel open source CFD-DEM for resolved particlefluid interaction. In: Proceedings of the $9^{\text {th }}$ International Conference on CFD in the Minerals and Process Industries. CSIRO, Melbourne, Australia; 10-12 December 2012 
Han, X., Wang, Q., Park, Y., T'Joen, C., Sommers, A., \& Jacobi, A. (2012). A review of metal foam and metal matrix composites for heat exchangers and heat sinks. Heat Transfer Engineering, 33(12), 991-1009.

Hilton, J. E., \& Cleary, P. W. (2013). Dust modelling using a combined CFD and discrete element formulation. International Journal for Numerical Methods in Fluids, 72(5), 528-549.

Hooman, K., Tamayol, A., \& Malayeri, M. R. (2012). Impact of particulate deposition on the thermohydraulic performance of metal foam heat exchangers: A simplified theoretical model. Journal of Heat Transfer, 134(9), 92601.

Huisseune, H., De Schampheleire, S., Ameel, B., \& De Paepe, M. (2015). Comparison of metal foam heat exchangers to a finned heat exchanger for low reynolds number applications. International Journal of Heat and Mass Transfer, 89, 1-9.

Hung, T., \& Yan, W. (2013). Thermal performance enhancement of microchannel heat sinks with sintered porous media. Numerical Heat Transfer; Part A: Applications, 63(9), 666-686.

Inamdar, H. V., Groll, E. A., Weibel, J. A., \& Garimella, S. V. (2016). Prediction of air-side particulate fouling of HVAC\&R heat exchangers. Applied Thermal Engineering, 104, 720-733.

Kamath, P., Balaji, C., \& Venkateshan, S. (2014). Heat transfer enhancement with discrete heat sources in a metal foam filled vertical channel. International Communications in Heat and Mass Transfer, 53, 180-184.

Kloss, C., Goniva, C., Hager, A., Amberger, S., \& Pirker, S. (2012). Models, algorithms and validation for opensource DEM and CFD-DEM. Progress in Computational Fluid Dynamics, An International Journal, 12(2-3), 140-152.

Krar, S., \& Gill, A. (2003). Exploring advanced manufacturing technologies. Industrial Press Inc.

Kuruneru, S., Sauret, E., Saha, S., \& Gu, Y. (2016). Numerical investigation of the temporal evolution of particulate fouling in metal foams for air-cooled heat exchangers. Applied Energy, 184, 531-547.

Kuruneru, S., Sauret, E., Saha, S., \& Gu, Y. (2017). A coupled finite volume \& discrete element method to examine particulate foulant transport in metal foam heat exchangers. International Journal of Heat and Mass Transfer, 115, 43-61.

Kuruneru, S., Sauret, E., Vafai, K., Saha, S., \& Gu, Y. (2017). Analysis of particle-laden fluid flows, tortuosity and particle-fluid behaviour in metal foam heat exchangers. Chemical Engineering Science, 172, 677-687.

Li, P., Fan, X., \& Chen, Z. (2016). Numerical study on the heat transfer of micro elliptic pin fins in a rectangular mini-channel. Numerical Heat Transfer, Part A: Applications, 70(11), 1242-1252.

Li, Y., Gong, L., Xu, M., \& Joshi, Y. (2017). Thermal performance analysis of biporous metal foam heat sink. Journal of Heat Transfer, 139(5), 052005-052005-8. 
Lotfizadeh, H., Mehrizi, A., Motlagh, M., \& Rezazadeh, S. (2015). Thermal performance of an innovative heat sink using metallic foams and aluminum nanoparticles-experimental study. International Communications in Heat and Mass Transfer, 66, 226-232.

Luo, K., Tan, J., Wang, Z., \& Fan, J. (2016). Particle resolved direct numerical simulation of gas solid dynamics in experimental fluidized beds. AlChE Journal, 62(6), 1917-1932.

Mahdi, H., Lopez, P., Fuentes, A., \& Jones, R. (2006). Thermal performance of aluminium-foam CPU heat exchangers. International Journal of Energy Research, 30(11), 851-860.

Moore, D. A. Characterization of fibre accumulation fouling in fine pitched heat sinks. In: Proceedings of the $25^{\text {th }}$ Annual IEEE Semiconductor Thermal Measurement and Management Symposium, San Jose, California, U.S.A.; 15-19 March 2009.

Mousel, J. A., \& Marshall, J. S. (2010). Aggregate growth and breakup in particulate suspension flow through a micro-nozzle. Microfluidics and Nanofluidics, 8(2), 171-186.

Mozdianfard, M. R., \& Behranvand, E. (2015). Fouling at post desalter and preflash drum heat exchangers of CDU preheat train. Applied Thermal Engineering, 89, 783-794.

Müller-Steinhagen, H., Malayeri, M. R., \& Watkinson, A. P. (2013). Heat exchanger fouling and cleaning2011. Heat Transfer Engineering, 34(8-9), 653-654.

Muyshondt, A., Nutter, D., \& Gordon, M. (1998). Investigation of a fin-and-tube surface as a contaminant sink. ASHRAE IAQ 1998, 207-211.

OpenFOAM v4.1. (2016). https://openfoam.org/release/4-1/

ParaView v4.1.0. (2014). https://blog.kitware.com/paraview-4-1-0-available-for-download/

Qian, F., Huang, N., Zhu, X., \& Lu, J. (2013). Numerical study of the gas-solid flow characteristic of fibrous media based on SEM using CFD-DEM. Powder Technology, 249, 63-70.

Rad, S. E., Afshin, H., \& Farhanieh, B. (2015). Heat transfer enhancement in shell-and-tube heat exchangers using porous media. Heat Transfer Engineering, 36(3), 262-277.

Ranut, P., Nobile, E., \& Mancini, L. (2014). High resolution microtomography-based CFD simulation of flow and heat transfer in aluminum metal foams. Applied Thermal Engineering, 69(1-2), 230-240.

Romaní Fernández, X., \& Nirschl, H. (2013). Simulation of particles and sediment behaviour in centrifugal field by coupling CFD and DEM. Chemical Engineering Science, 94, 7-19.

Sauret, E., Abdi, I., Hooman, K. (2014). Fouling of waste heat recovery: numerical and experimental results. In: Proceedings of the $19^{\text {th }}$ Australasian Fluid Mechanics Conference, Melbourne, Australia.; 8-11 December 2014. 
Sauret, E., \& Hooman, K. (2014). Particle size distribution effects on preferential deposition areas in metal foam wrapped tube bundle. International Journal of Heat and Mass Transfer, 79, 905-915.

Seyf, H. R., \& Layeghi, M. (2010). Numerical analysis of convective heat transfer from an elliptic pin fin heat sink with and without metal foam insert. Journal of Heat Transfer, 132(7), 1-9.

Schampheleire, S., Jaeger, P., Huisseune, H., Ameel, B., T'Joen, C., Kerpel, K., \& Paepe, M. (2013). Thermal hydraulic performance of 10 PPI aluminium foam as alternative for louvered fins in an HVAC heat exchanger. Applied Thermal Engineering, 51(1), 371-382.

Schoenitz, M., Grundemann, L., Augustin, W., \& Scholl, S. (2015). Fouling in microstructured devices: A review. Chemical Communications, 51(39), 8213-8228.

Siegel, J. A. (2002). Particulate fouling of HVAC heat exchangers. (Doctoral dissertation). Retrieved from https://www.camfil.com/FileArchive/_10_Camfil_Message_CamTab/Air\%20Quality/Particle\%20Fouling\% 20of\%20Heat\%20Exchangers.pdf

Siegel, J. A., \& Nazaroff, W. W. (2003). Predicting particle deposition on HVAC heat exchangers. Atmospheric Environment, 37(39), 5587-5596.

Tamayol, A., \& Bahrami, M. (2011). Transverse permeability of fibrous porous media. Physical Review E Statistical, Nonlinear, and Soft Matter Physics, 83(4), 046314.

Tongcai, W., Luan, W., Wang, W., \& Tu, S. (2014). Waste heat recovery through plate heat exchanger based thermoelectric generator system. Applied Energy, 136, 860-865.

Traore, P., Laurentie, J., Dascalescu, L. (2015). An efficient 4 way coupling CFD-DEM model for dense gas-solid particulate flows simulations. Computers \& Fluids, 113, 65-76.

Tsuji, Y., Tanaka, T., \& Ishida, T. (1992). Lagrangian numerical simulation of plug flow of cohesionless particles in a horizontal pipe. Powder Technology, 71(3), 239-250.

Tu, J., Yeoh, G., \& Liu, C. (2012). Computational Fluid Dynamics: A Practical Approach. ButterworthHeinemann.

Visser, J., \& Jeurnink, T. J. M. (1997). Fouling of heat exchangers in the dairy industry. Experimental Thermal and Fluid Science, 14(4), 407-424.

Wang, F., He, Y., Tong, Z., \& Tang, S. (2017). Real-time fouling characteristics of a typical heat exchanger used in the waste heat recovery systems. International Journal of Heat and Mass Transfer, 104, 774-786.

Xu, H. J., Qu, Z. G., \& Tao, W. Q. (2011). Thermal transport analysis in parallel-plate channel filled with open-celled metallic foams. International Communications in Heat and Mass Transfer, 38(7), 868-873.

Yang, K., Chung, C., Lee, M., Chiang, S., Wong, C., \& Wang, C. (2013). An experimental study on the heat dissipation of LED lighting module using metal/carbon foam. International Communications in Heat and Mass Transfer, 48, 73-79. 
Yang, Y., Tsai, K., Tang, H., \& Chung, S. (2016). Numerical simulations and optimization of porous pin fins in a rectangular channel. Numerical Heat Transfer, Part A: Applications, 70(7), 791-808.

Yang, M., Young, A., Niyetkaliyev, A., \& Crittenden, B. (2012). Modelling fouling induction periods. International Journal of Thermal Sciences, 51(1), 175-183.

Yucel, N., \& Guven, R.T. (2007). Forced-convection cooling enhancement of heated elements in a parallelplate channels using porous inserts. Numerical Heat Transfer, Part A: Applications, 51(3), 293-312. 